\title{
SERUM TRANSAMINASE VARIATIONS IN CHILDHOOD
}

\author{
BY \\ PAOLO TOLENTINO and MARCELLA ROSSI \\ From the Departments of Infectious Diseases and Paediatrics, University of Genoa
}

(RECEIVED FOR PUBLICATION MARCH 10, 1958)

The study of serum transaminases has recently entered clinical practice. In December, 1954, Wróblewsky and La Due demonstrated an increase of glutamic-oxalacetic (GOT) transaminase in the serum of patients suffering from various liver diseases and in carbon tetrachloride intoxication. In 1955, de Ritis, Coltorti and Giusti demonstrated an increase of both aspartic-ketoglutaric (GOT) and alanine-ketoglutaric (GPT) transaminases in the serum of patients with infectious hepatitis. This increase was accompanied by a remarkable inversion of the ratio between aspartic-ketoglutaric (GOT) and alanine-ketoglutaric activity (GPT), ( $\frac{\text { GOT }}{\text { GPT }}$ ) which stands above unity in healthy people and notably below in infectious hepatitis. While the increase of serum transaminase is generally ascribed to extensive necrosis of the hepatic cells and the consequent passage into the blood of cellular enzymes, the inversion of the ratio has not yet been satisfactorily explained.

A series of clinical and experimental observations made during the last two years has enabled us to reach some interesting conclusions. Our clinical researches concerned about 100 cases of acute infectious diseases, including infectious hepatitis, rheumatic fever, measles, scarlet fever, whooping cough, chickenpox, infectious mononucleosis, mumps and poliomyelitis. In some of the abovementioned diseases multiplication of the causal agent in the liver during the incubation stage takes place, and liver damage is frequently observed at needle-biopsy.

In estimating the serum transaminases we used the method of Tonhazy, White and Umbreit (1950) reading with Pulfrich's photometer, filter S 53. The results are set out in Figs. 1, 2 and 3, the values being expressed in micromoles of pyruvic acid.

These figures show that only in infectious hepatitis, from virus $\mathbf{A}$ or $\mathbf{B}$, is there a definite increase of both transaminases accompanied by the characteristic inversion of the ratio. This specific finding is probably related to the extensive liver

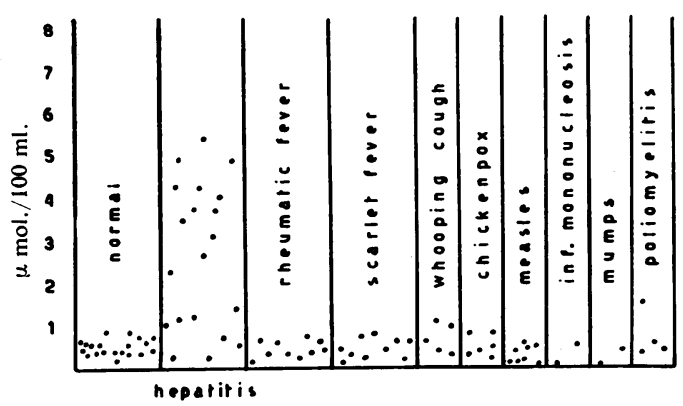

FIG. 1.-Serum aspartic-ketoglutaric (GOT) transaminase.

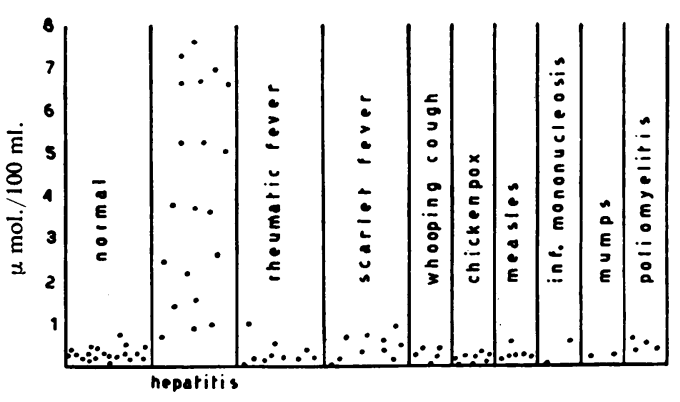

Fig. 2.-Serum alanine-ketoglutaric (GPT) transaminase.

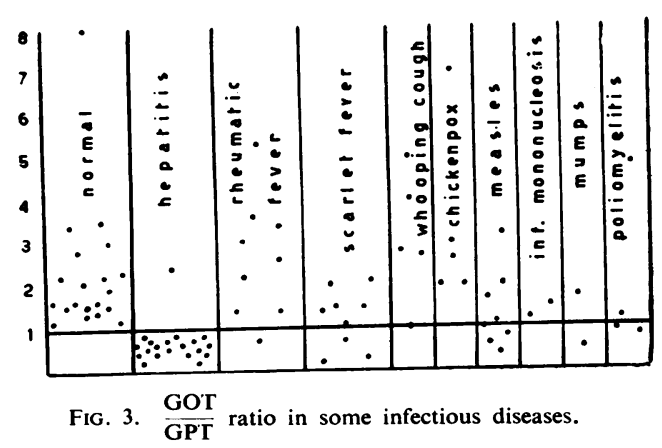


necrosis that is present only in this disease. However, we found the ratio inverted without a considerable increase of transaminase in a certain percentage of cases in other infectious diseases; we never found inversion in healthy subjects (Tolentino and Rossi, 1957a).

In numerous cases of hepatitis we estimated the serum transaminases throughout the course of the disease and observed that the values decreased with clinical improvement and rose again during relapses. The last pathological finding to disappear was the inversion of the GOT/GPT ratio.

In some cases we were also fortunate enough to be able to show that the increase in serum transaminases occurred very early, appearing about a month before the clinical manifestations. We observed this in children from a school, who were in contact with hepatitis patients. Fig. 4 charts the course of one such case and demonstrates that the

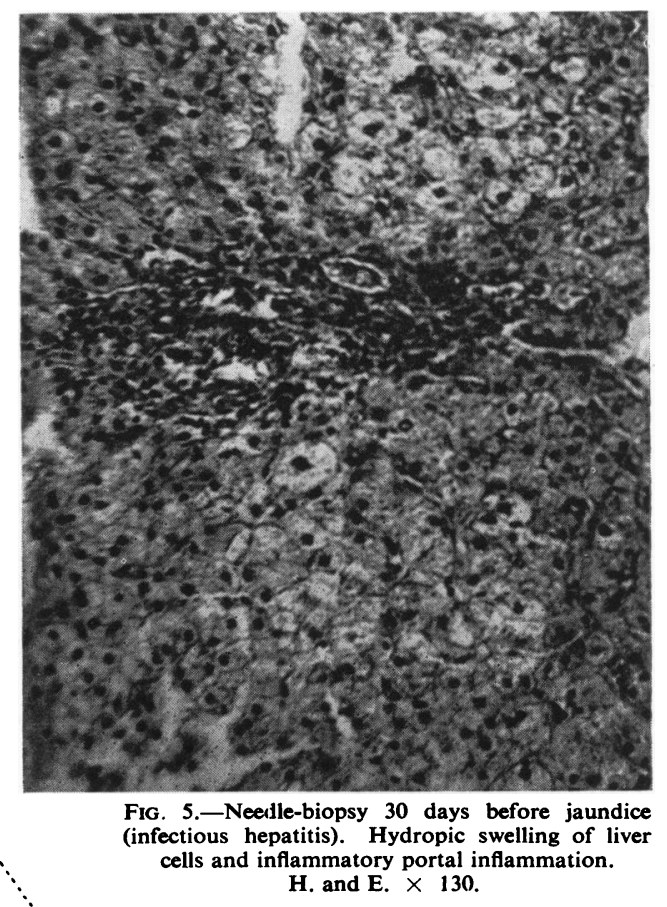

pathological increase of transaminases, with inversion of the ratio, appeared as early as 36 days before fever and jaundice. The estimation of transaminases is, therefore, a valuable aid in detecting cases of hepatitis just at the beginning of the incubation period, with obvious prophylactic advantages. Thus it should be used routinely in blood donors in order to eliminate those with pathological values (Tolentino and Rossi, 1957b).

Since at the beginning of the incubation period there is sufficient necrotic damage of the hepatic cells to induce an increase of transaminases, and to be manifest at biopsy (as demonstrated in one case in Fig. 5), it seems that even at this early stage the virus is exerting its injurious action upon the liver. The unusually long duration of the incubation stage could be due to a peculiar slowness of virus multiplication, and clinical signs would appear only when a sufficient portion of tissue had been destroyed.

As has been mentioned above a dissociation exists in some clinical cases between increase of the
Methods: Transaminase: Tonhazy-White-Umbreit. Bilirubinaemia: Jendrassik Czike. Phosphatase: King-Armstrong. Cholinesterase: Stedman-Lucas. Electrophoresis on paper. 
transaminases and inversion of the ratio: on the one hand some infectious diseases show an inversion of the GOT/GPT ratio without an increase of values, while on the other hand obstructive (as opposed to infective) jaundice demonstrates increased values without inversion (cases of obstructive jaundice of the newborn) (Kove, Goldstein and Wróblewski, 1957; some cases of ours). We sought an explanation of these phenomena in animal experiments.

This research gave the following results:

(1) In guinea pigs bile-duct ligature produced after only one hour a very quick and remarkable increase (about 10 times) of asparticketoglutaric (GOT) transaminase and

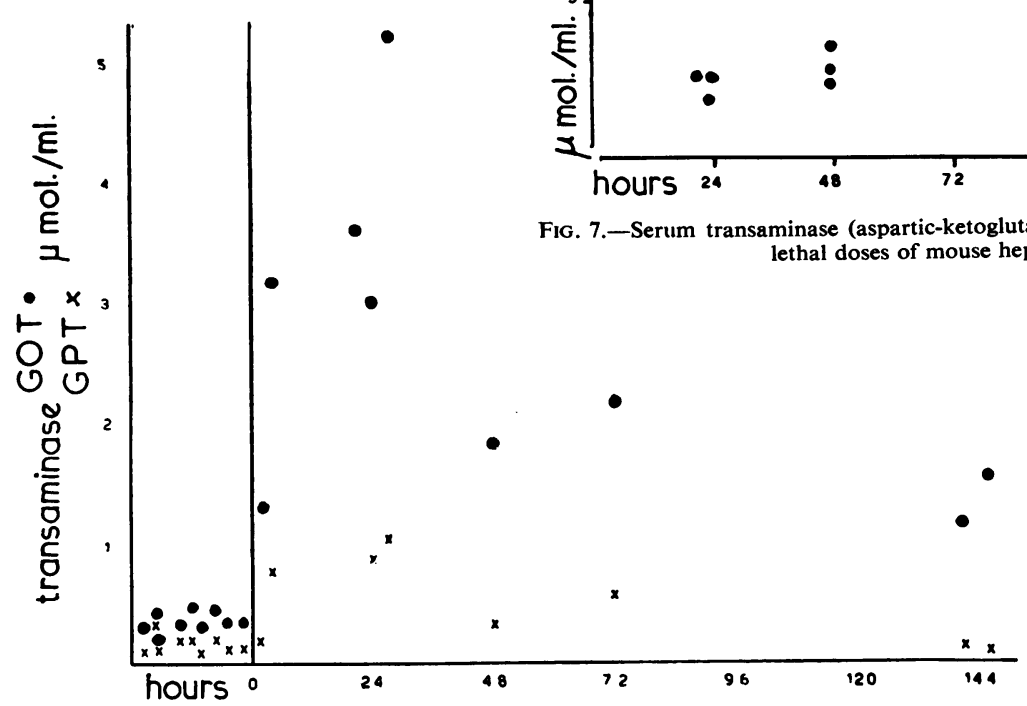

FIG. 6.-Serum transaminases in guinea pigs before and after ligature of the bile duct.

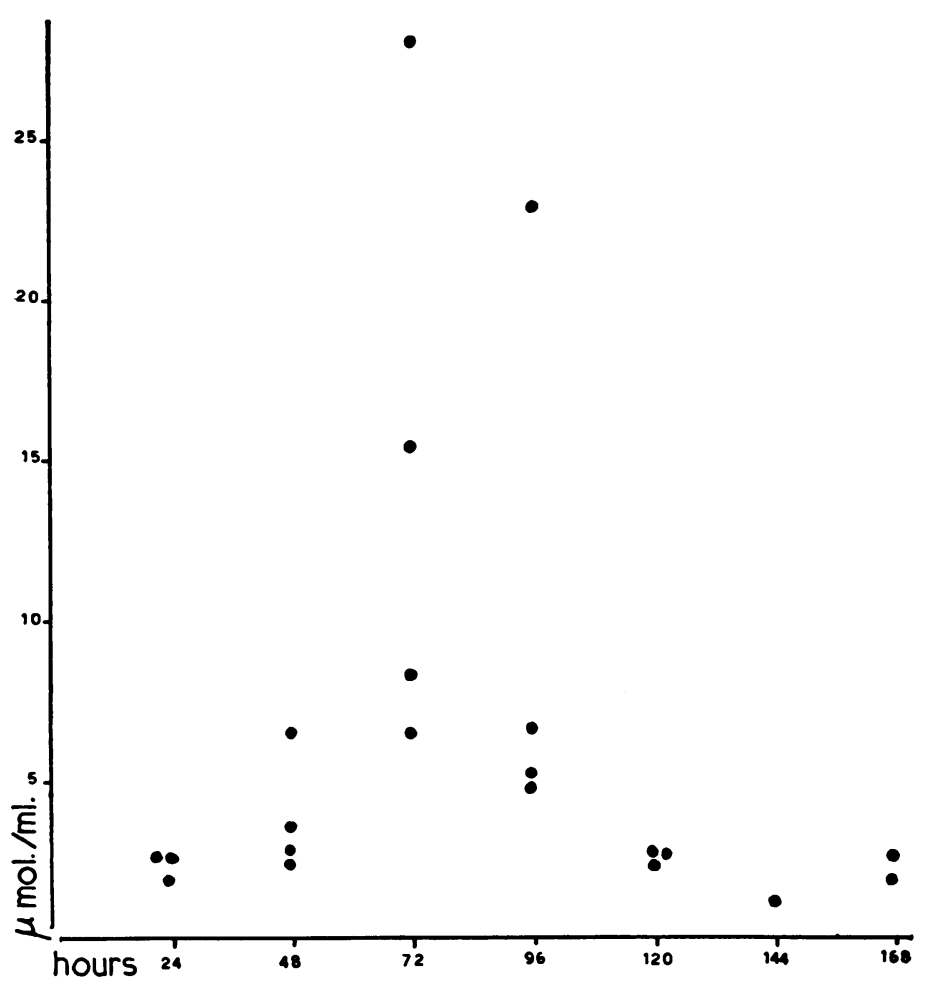

FIG. 7-Serum transaminase (aspartic-ketoglutaric (GOT)) in mice inoculated with sublethal doses of mouse hepatitis virus $\left(\mathrm{MHV}_{\mathbf{3}}\right)$.

a smaller increase of alanineketoglutaric (GPT) transaminase; the normal ratio (Fig. 6) was therefore maintained.

(2) In mice infected with mouse hepatitis by virus $\mathrm{MHV}_{3}$ there was an increase of serum transaminases just as in human infectious hepatitis (Fig. 7), and this increase was preceded by a similar inversion of the ratio in the mitochondria of the damaged liver (Tolentino, Nordio and Rossi, 1957).

TABLE 1

VALUES OF LIVER TRANSAMINASES IN NORMAL AND IN TYPHOID-VACCINATED GUINEA-PIGS

\begin{tabular}{|c|c|c|c|c|c|c|}
\hline & & Normal & & & Vaccinated & \\
\hline Transaminase Activity & GOT & GPT & $\begin{array}{c}\text { Ratio } \\
\text { GOT/GPT }\end{array}$ & GOT & GPT & $\begin{array}{c}\text { Ratio } \\
\text { GOT/GP T }\end{array}$ \\
\hline Liver homogenate ( $\mu \mathrm{mol} . / 100 \mathrm{mg})$. & $44 \cdot 14$ & $6 \cdot 85$ & 6.85 & $51 \cdot 30$ & $13 \cdot 26$ & 4.27 \\
\hline Mitochondria ( $\mu \mathrm{mol} . / \mathrm{mg} . \mathrm{N}$ ) & $9 \cdot 78$ & $1 \cdot 14$ & 8.48 & $9 \cdot 00$ & $3 \cdot 66$ & 2.90 \\
\hline Supernatant phase ( $\mu \mathrm{mol} . / \mathrm{mg}$. N.) $\ldots$ & $7 \cdot 96$ & $1 \cdot 29$ & 9.47 & $7 \cdot 55$ & $2 \cdot 14$ & 4.26 \\
\hline
\end{tabular}


(3) In guinea-pigs immunized by typhoid vaccine an increase of alanine-ketoglutaric (GPT) transaminase in the mitochondria and in the supernatant fluid occurred with an alteration of the GOT/GPT ratio in favour of alanine-ketoglutaric (GPT) transaminase (Table 1) (Tolentino, 1957).

Previous research in our department had demonstrated that antigenic stimulation, such as provided by vaccination or infectious diseases, produces a hydropic swelling of the hepatic cells related to their increased function. The simultaneous internal displacement of transaminases in the cell may be responsible for the inversion of the serum transaminases (without a significant increase) in some infectious diseases. In infectious hepatitis the phases succeed each other: there is first, hydropic swelling with internal displacement of enzymes, followed by cellular necrosis and a consequent passage of cellular enzymes into the blood. In obstructive necrosis, produced by bile duct ligature, on the contrary, the first phase, the internal displacement of the enzymes, is absent and therefore there is no inversion of the ratio in the blood.

\section{REFERENCES}

Kove, S., Goldstein, S. and Wróblewski, F. (1957). Pediatrics, 20,

Ritis, F. de, Coltorti, M. and Giusti, G. (1955). Minerva Med. (Torino), 46, 1207.

Tolentino, P. (1957). Intern. Conference of Hepatol. Perugia.

Nordio, S. and Rossi, M. (1956). Boll. Soc. ital. Biol. sper.,

32, 780.
and Rossi, M. (1957a). Minerva pediat. (Torino), 9, 377.

- and Rossi, M. (1957a). Minerva pediat. Tonhazy, N. E., White, N. G. and Umbreit, W. W. (1950). Arch.
Biochem., 28, 36.

Wróblewski, F. and La Due, J. S. (1954). J Lab. clin. Med., 44, 958. 